\title{
Drug susceptibility and molecular epidemiology of Escherichia coli in bloodstream infections in Shanxi, China
}

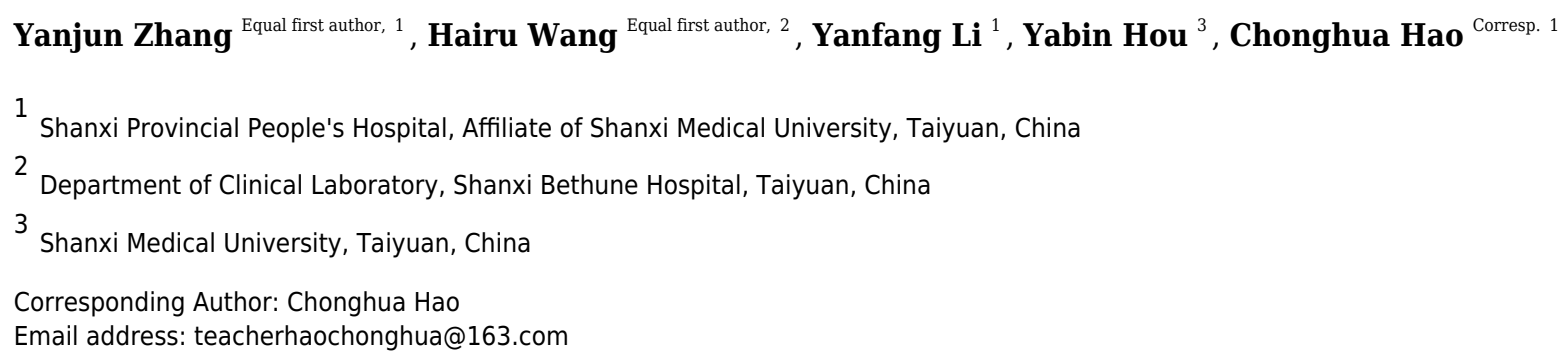

Objectives: We carried out a retrospective study to investigate the drug susceptibility and genetic relationship of clinical $E$. coli isolates from patients with BSIs in Shanxi, China. Methods: E. coli isolates causing BSIs were consecutively collected from June 2019 to March 2020. Antimicrobial susceptibility testing was performed by broth microdilution method. PCR was used to detect antimicrobial resistance genes coding for extendedspectrum $\beta$-lactamases (ESBLS), phylogenetic groups and seven housekeeping genes of $E$. coli. Results: A total of $76 \mathrm{E}$. coli were collected. Antimicrobial susceptibility testing revealed that the top $6 \mathrm{E}$. coli resistant antibiotics were ampicillin $(90.7 \%)$, ciprofloxacin (69.7\%), cefazolin (65.7\%), levofloxacin (63.1\%), ceftriaxone and cefotaxime $(56.5 \%)$. Among the 76 isolates, 43 produced ESBLs. Molecular analysis showed that CTX-M-14 was the most common ESBLs, followed by CTX-M-15 and CTX-M-55. Phylogenetic group D (42.2\%) predominated, followed by group B2 (34.2\%), group A (18.4\%) and group B1 (5.2\%). The most prevalent sequence types (STs) were ST131 (15/76), ST69 (12/76) and ST38 (6/76). Conclusions: This study is the first to report the phenotypic and molecular characteristics of $E$. coli isolated from BSIs in Shanxi, China. Our results indicated a high prevalence of MDR in E. coli strains isolated from BSIs and a serious spread of ESBL genes in Shanxi, especially the epidemiological bla ${ }_{\mathrm{CTX}-\mathrm{M}}$. Phylogenetic analysis indicated genetic diversity among $E$. coli BSIs isolates. 


\section{Drug susceptibility and molecular epidemiology of} 2 Escherichia coli in bloodstream infections in Shanxi,

3 China

4 Yanjun Zhang ${ }^{1}$, Hairu Wang ${ }^{2}$, Yanfang $\mathrm{Li}^{1}$, Yabin $\mathrm{Hou}^{3}$, Chonghua Hao ${ }^{1}$

6 'Department of Clinical Laboratory, Shanxi Provincial People's Hospital, Affiliate of Shanxi

7 Medical University, Taiyuan, Shanxi Province, China

$8 \quad 2$ Department of Clinical Laboratory, Shanxi Bethune Hospital, Taiyuan, Shanxi Province, China

$9 \quad{ }^{3}$ Department of Clinical laboratory diagnostics, Shanxi Medical University, Taiyuan, Shanxi

10 Province, China

Corresponding Author:

Chonghua Hao ${ }^{1}$

Shuangtasi Street, Taiyuan City, Shanxi Province, 030012, China

Email address: teacherhaochonghua@163.com

\section{Abstract}

Objectives: We carried out a retrospective study to investigate the drug susceptibility and genetic relationship of clinical E. coli isolates from patients with BSIs in Shanxi, China.

Methods: E. coli isolates causing BSIs were consecutively collected from June 2019 to March 2020. Antimicrobial susceptibility testing was performed by broth microdilution method. PCR was used to detect antimicrobial resistance genes coding for extended-spectrum $\beta$-lactamases (ESBLs), phylogenetic groups and seven housekeeping genes of E. coli. top 6 E. coli resistant antibiotics were ampicillin (90.7\%), ciprofloxacin (69.7\%), cefazolin (65.7\%), levofloxacin (63.1\%), ceftriaxone and cefotaxime (56.5\%). Among the 76 isolates, 43 produced ESBLs. Molecular analysis showed that CTX-M-14 was the most common ESBLs, 
31 Conclusions: This study is the first to report the phenotypic and molecular characteristics of $E$.

32 coli isolated from BSIs in Shanxi, China. Our results indicated a high prevalence of MDR in $E$. 33 coli strains isolated from BSIs and a serious spread of ESBL genes in Shanxi, especially the 34 epidemiological $b l a_{\mathrm{CTX}-\mathrm{M}}$. Phylogenetic analysis indicated genetic diversity among E. coli BSIs 35 isolates.

36 Keywords: Escherichia coli, drug susceptibility, bloodstream infections, molecular 37 epidemiology, Extended-spectrum beta-lactamases (ESBLs)

\section{Introduction}

39

40

41

42

43

44

45

46

47

48

49

50

51

52

53

54

55

56

57

58

59

60

61

62

63

64

65

66

Bloodstream infections (BSIs), one of the most common severe infections, is also an important complication leading to extended hospitalization and increased mortality (Yoon et al. 2018). BSIs is occurred in about 2 million episodes and is responsible for about 250000 deaths in United States and Europe, ranking among the top seven causes of death in North America and Europe (Goto \& Al-Hasan 2013; Sader et al. 2019).

In recent years, the main etiology of BSIs has been patterns of antimicrobial resistance, especially with Gram-negative bacteria (Sader et al. 2019). Escherichia coli (E. coli) is an important causative pathogen of Gram-negative BSIs, whether in view of healthcare-associated - infection surveillance or antimicrobial resistance in modern global health (Kern \& Rieg 2019; Tsuzuki et al. 2020; Xiao et al. 2019a). The incidence of BSIs caused by E. coli has increased in Europe, with an annual increase of 6\% between April 2012 and March 2014 (Bou-Antoun et al. 2016). Data from the SENTRY Antimicrobial Surveillance Program showed that the proportion of $E$. coli in all BSIs isolates increased from $18.7 \%$ in $1997-2000$, to $24.0 \%$ in $2013-$ 2016 (Diekema et al. 2019). The most frequently organism identified from blood samples, was $E$. coli $(23.1 \%)$ according to the statistical results from the China antimicrobial surveillance network in 2018 (Hu et al. 2019).

The 30-day all-cause mortality rate after E. coli BSIs is about $16 \%$, which may increase with the rises of antimicrobial resistance (Vihta et al. 2018). Extended-spectrum $\beta$-lactamases (ESBLs) production is the main drug resistance mechanism identified in E. coli (Nepal et al. 2017). Unfortunately, Lei Tian et al, found that the proportion of $E$. coli producing ESBLs increased significantly from $0 \%$ in $1998-2002$ to $76.2 \%$ in 2008-2012 in China (Tian et al. 2019). The increase of the acquired multidrug resistance rate of ESBLs-producing E. coli has placed significant restrictions on appropriate and reliable antimicrobial therapeutic options (Bi et al. 2017). Timely administration of appropriate empiric antimicrobial therapy can save lives, but the most appropriate empiric antimicrobial regimen requires a full understanding of the common causes of BSIs and its drug resistance patterns (Marchello et al. 2019). Data on full-scale susceptibility surveillance and molecular epidemiological investigation of $E$. coli causing BSIs in Shanxi Province of China are lacking. In this article, we set out to analyze the susceptibility of 
67 antimicrobial agents, distribution of drug resistance genes, genetic relationship and sequence 68 types (ST) of E. coli causing BSIs in Shanxi, China.

\section{Materials \& Methods}

\section{Setting and study design}

This retrospective study was conducted in a general teaching hospital affiliated to Shanxi Medical University (Shanxi Provincial People's Hospital) in Taiyuan, the capital city of Shanxi Province in North China. Shanxi Provincial People's Hospital is a comprehensive tertiary hospital, with 2800 beds and a total of approximately 1.28 million patients annually. The hospital provides better medical services to the people of Shanxi Province, about 370,000 people every year.

The study was approved by the Ethical Committee of Shanxi Provincial People's Hospital, Shanxi Medical University (Ethical Application Ref: 2020-7). Written informed consent was obtained from participants for our study.

\section{Bacterial Isolates}

We performed a retrospective cross-sectional study of patients with $E$. coli BSIs between June 2019 and March 2020. Cases of E. coli BSIs were identified from the laboratory database in the Department of Clinical Laboratory, Shanxi Provincial People's Hospital. Each patient was included only once, if multiple blood cultures from the same patient were positive, only the first episode was reviewed and recorded. All isolates were identified by matrix-assisted laser desorption ionization-time of flight mass spectrometer (bioMérieux, Marcy-l'Étoile, France) and stored in broth containing $30 \%$ glycerol at $-80^{\circ} \mathrm{C}$ until further experiments.

\section{Antimicrobial Susceptibility Testing and Detection of ESBLs and Carbapenemases}

Antimicrobial susceptibility testing was carried out by broth microdilution method according to Clinical and Laboratory Standard Institute (CLSI) recommendations (CLSI, 2019) for ampicillin, amoxicillin-clavulanate, piperacillin-tazobactam, cefazolin, cefoxitin, ceftriaxone, cefepime, cefotaxime, cefoperazone-sulbactam, aztreonam, ertapenem, imipenem, meropenem, amikacin, gentamicin, tobramycin, ciprofloxacin, levofloxacin, tigecycline and trimethoprimsulfamethoxazole. The breakpoint of tigecycline was based on European Committee on Antimicrobial Susceptibility Testing (EUCAST) (EUCAST, 2019), other drugs were interpreted by CLSI and cefoperazone/sulbactam was referred to cefoperazone in CLSI (CLSI, 2019). E. coli ATCC 25922 and ATCC 35218 were used as quality control strains for antimicrobial susceptibility testing.

Broth dilution test (cefotaxime and cefotaxime-clavulanic acid, ceftazidime and ceftazidime-clavulanic acid) was used as confirmatory test for ESBLs producers following CLSI 
101

102

103

104

105

106

107

108

109

110

111

112

113

114

115

116

117

118

119

120

121

122

123

124

125

126

127

128

129

130

131

\section{2}

133 134

criteria (CLSI, 2019). Either cefotaxime or cefotadime combined with clavulanic acid, the MIC was decreased by threefold concentration, which can be considered as ESBLs positive. Klebsiella pneumoniae ATCC 700603 was used as positive control for ESBLs production. According to the susceptibility results, the ESBLs genes were further detected in the positive isolates.

\section{Detection of Resistance Genes}

Template DNA was extracted by boiling method as mentioned in previous article (Zhao et al. 2015). Polymerase chain reaction (PCR) were performed to screen for bla $a_{\mathrm{TEM}}$, bla $_{\mathrm{SHV}}$, bla $_{\mathrm{CTX}}$

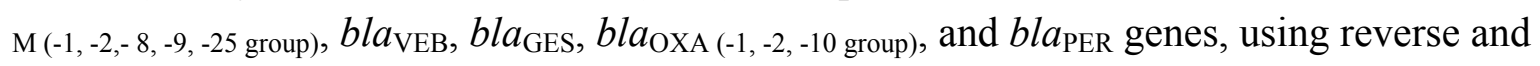
forward primers pairs listed in Table 1, as previously described ( Wang et al. 2016). Positive amplicons were sequenced using ABI3730xIDNAAnalyzer by Sangon Biotech (Shanghai, China) and the DNA sequences were compared in GenBank (http://blast.ncbi.nlm.nih.gov) to identify the allelic variant.

\section{Phylogenetic group analysis and multilocus sequence typing}

According to the well recognized phylogenetic grouping protocol proposed by Clermont et al. (Clermont et al. 2000), four major phylogenetic groups (A, B1, B2 and D) in the E. coli isolates were determined using the method of triple PCR based on three genetic markers, namely chuA, yjaA and TspE4.C2. chuA encodes outer membrane hemin receptor gene that involves in heme transport. yjaA encodes for gene responsible for cellular response to hydrogen peroxide and acid stress and TspE4.C2 DNA encodes for putative lipase esterase gene (Javed et al. 2021). PCR was performed to determine the seven conserved housekeeping genes (adk, fumC, gyr $B, i c d$, $m d h$, purA, and recA) of E. coli, and the primer pairs were listed in Table 2. The allelic profiles and STs were described by the combination of the 7 alleles on line (http://mlst.warwick.ac.uk/mlst/dbs/Ecoli/).

\section{Statistical analysis}

The statistical analysis was performed using SPSS 25.0 (IBM, Armonk, NY, USA). When fitting a normal distribution, we presented continuous numerical variables as mean and standard deviation (SD). For categorical variables, results were expressed as the percentages of the groups from which they were derived. The $\chi^{2}$ test was used to compare categorical variables. All tests were two-sided, and $p<0.05$ was considered statistically significant.

\section{Results}

\section{Characteristics of Total Patient Population}

A total of 76 eligible $E$. coli isolates were enrolled during the study period. From the total of 76 E. coli causing BSIs they were isolates from females $(42 / 76)$ and $(34 / 76)$ from males. The age 
135 of patients ranged from 13 to 90 years and the mean age was $62.1 \pm 14.9$ years. The mean age did

136

137

138

139

140

141

142

143

144

145

146

147

148

149

150

151

152

153

154

155

156

157

158

159

160

161

162

163

164

165

166

167

168

169

not differ significantly in ESBLs-producing isolates compared to non-ESBLs-producing E. coli.

\section{Antimicrobial Susceptibility Tests}

Antimicrobial susceptibility testing revealed that the top 6 E. coli resistant antibiotics were ampicillin (90.7\%), ciprofloxacin (69.7\%), cefazolin (65.7\%), levofloxacin (63.1\%), ceftriaxone and cefotaxime (56.5\%). On the contrary, carbapenems, piperacillin-tazobactam, amikacin and tigecycline exhibited excellent activity against E.coli isolates in vitro with susceptibility up to $100.0 \%$. Of the 76 isolates, $56.5 \%$ were confirmed as ESBLs-producing E. coli. Compared with ESBLs-producing $E$. coli, the non-ESBLs-producing $E$. coli showed higher susceptibility to cefazolin, ceftriaxone, cefepime, cefotaxime, aztreonam $(P<0.05)$ (Table 3$)$. Despite the significant differences, both ESBLs-producing and non-ESBLs-producing $E$. coli showed lower susceptibility to ampicillin, ciprofloxacin, levofloxacin, trimethoprim-sulfamethoxazole and gentamicin. Out of total $76 \mathrm{E}$. coli isolates, 56 (73.6\%) were multidrug resistant (MDR) (nonsusceptibility to $\geq 1$ agent in $\geq 3$ antimicrobial categories) isolates. The rates of MDR isolates were $97.6 \%$ and $42.4 \%$ in ESBL-producing and non-ESBL-producing E. coli, respectively.

\section{Characterization of Resistance genes}

A total of 71 drug-resistant genes including 28 bla $_{\mathrm{CTX-M-14}}, 20$ bla $_{\mathrm{TEM}-1}, 10$ bla $_{\mathrm{CTX}-\mathrm{M}-15}, 7$ $b l a_{\mathrm{OXA}-1}$ and 6 bla $a_{\mathrm{CTX}-\mathrm{M}-55}$ were identified among the 43 ESBLs-producing isolates, where they have one, two and three ESBLs genes in 19 isolates 20 and 4 isolates respectively. The highest detection rate was $b l a_{\mathrm{CTX}-\mathrm{M}-14}(39.4 \%, 28 / 71)$, followed by bla $a_{\mathrm{TEM}-1}(28.2 \%, 20 / 71), b_{\text {CTX-M-15 }}$

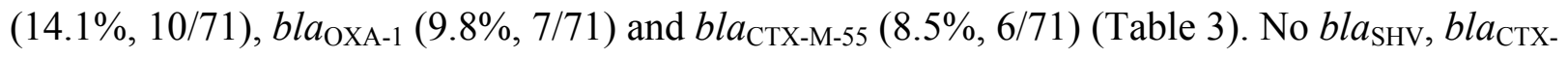
$\mathrm{M}(-2,-8,-25$ group $), b l a_{\mathrm{GES}}, b l a_{\mathrm{VEB}}, b l a_{\mathrm{OXA}(-2,-10 \text { group })}$ or $b l a_{\mathrm{PER}}$ genes were found.

\section{Phylogenetic characterization}

The phylogenetic lineages were determined in the 76 isolates: phylogenetic group D (42.2\%) predominated, followed by group B2 (34.2\%), group A (18.4\%) and group B1 (5.2\%). Among the 43 ESBLs-producers, phylogenetic group D (41.8\%) predominated, followed by group B2 (37.3\%), A (16.2\%) and B1 (4.7\%). On the other side, among the 33 non-ESBLsproducers, phylogenetic group D (42.4\%) predominated, followed by group B2 (30.3\%), A $(21.2 \%)$ and B1 (6.1\%). The percentage of phylo-groups identified were the same regardless of

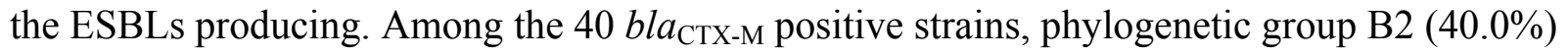
predominated, followed by group D (37.5\%), group A (17.5\%) and group B1 (5.0\%) (Figure 1).

\section{Multilocus Sequence Typing}

Among the 76 isolates, a total of 28 different sequence types (STs) were identified in this study (Table 4). The ST131 was the most frequently ST identified (19.7\%, 15/76), followed by ST69 (15.7\%, 12/76), ST38 (7.8\%, 6/76), ST1193 (6.5\%, 5/76), ST648 (5.2\%, 4/76), ST73

Peer] reviewing PDF | (2021:04:59823:2:0:NEW 30 Sep 2021) 
$170(5.2 \%, 4 / 76)$, ST46 (3.9\%, 3/76), ST405 (3.9\%, 3/76) and other uncommon STs that were

171

172

173

174

175

176

177

\section{Discussion}

179

180

181

182

183

184

185

186

187

188

189

190

191

192

193

194

195

196

197

198

199

200

201

202

203

204

205

206

207 2017).
The increased consumption of antimicrobial agents, the high prevalence and dissemination of drug resistance genes in pathogens, and the poor prevention and control strategies for infections lead to the increase of antimicrobial resistance (Parajuli et al. 2017). Approximately 700,000 deaths in antimicrobial resistance every year in the world, which is expected to soar to a staggering 10 million in 2050 (Huh et al. 2020). As pathogens'antimicrobial resistance rates and mortality in BSI patients increase, monitoring of microorganisms and antimicrobial resistance has become critical (Mehl et al. 2017). Our study not only monitored the resistance phenotype of E. coli, but also detected the distribution of drug resistance genes and the genetic relationship of the isolates, which provided the basis for designing strategies for the treatment and prevention of these serious infections.

The present study showed that resistance of several commonly used antibiotics used to treat BSIs in our area, such as penicillins, cefazolin, ceftriaxone, cefotaxime, fluoroquinolones and folate pathway inhibitors, was high (Table 3), which was consistent with previous report (Zhao et al. 2015). Fortunately, carbapenems, piperacillin-tazobactam, amikacin and tigecycline have low drug resistance rates, which should be considered for empirical treatment of $E$. coli isolates in our region. In this study, MDR accounted for $73.6 \%$ in E. coli causing BSIs, the value is lower than the result (85.6\%) of another similar study in China by Jie Ma et al (Ma et al. 2017), but much higher than that in E. coli causing pyelonephritis (40\%), sepsis (32\%) and skin and soft tissue infections (26\%) (Ranjan et al. 2017). Our study showed that among the 43 ESBLsproducing $E$. coli, $97.7 \%$ were MDR, while among 33 non-ESBLs-producing, only $42.4 \%$ were MDR. Since the ESBLs genes are usually found in large plasmids which also contain other antimicrobial resistant genes, most ESBLs producing organisms are MDR isolates (Ma et al.

In this study, the proportion of ESBLs-producing $E$. coli is $56.5 \%$, which was consistent with that in Zhejiang (57.6\%) (Xiao et al. 2019b), but much higher than in Japan (26.1\%), Vietnam (39.3\%), Singapore (33\%), and Brazil (12.8\%) (Hung et al. 2019; Komatsu et al. 2018). Since the 2000s, CTX-M have replaced TEM as the most common type of ESBLs in many countries (Liao et al. 2017; Zou et al. 2019). In the present study, genotypes showed remarkable increase in the CTX-M (40/71) compared to TEM (20/71) in ESBLs-producing E. coli. It is 
208

209

210

211

212

213

214

215

216

217

218

219

220

221

222

223

224

225

226

227

228

229

230

231

232

233

234

235

236

237

238

239

240

241

242

noteworthy that $b l a_{\text {CTX-M }}$ were strongly associated with ST131 (80\% carried $\left.b l a_{\text {CTX-M }}\right)$. We found that $b l a_{\mathrm{CTX}-\mathrm{M}-14}$ was the predominant $b l a_{\mathrm{CTX}-\mathrm{M}}$, followed by $b l a_{\mathrm{CTX}-\mathrm{M}-15}$ and $b l a_{\mathrm{CTX}-\mathrm{M}-55}$, while in Germany, bla $a_{\mathrm{CTX}-\mathrm{M}-15}$ was the most common and $b l a_{\mathrm{CTX}-\mathrm{M}-14}$ was the second most frequently identified genotype (Rohde et al. 2020). CTX-M-55, a variant of CTX-M-15 that contains a substitution of $\mathrm{A} 80 \mathrm{~V}$ within the $\beta$-lactamase possessing enhanced cephalosporin-hydrolyzing activity, was rarely found in clinical isolates previously (Liao et al. 2017; Zhang et al. 2019). While, in some surveys, the prevalence of bla $a_{\mathrm{CTX}-\mathrm{M}-55}$ has surpassed bla $_{\mathrm{CTX-M-15}}$ and become the secondary genotype of $b l a_{\text {СтХ-м }}$ (Hu et al. 2018; Zhao et al. 2015). It was observed that the

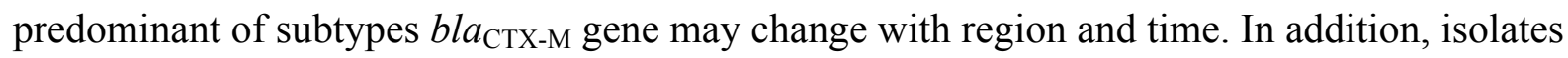
producing $b l a_{\text {OXA-1 }}$ also accounted for a larger proportion among the ESBLs-producers and mainly belonged to B2-ST131.

In this study we identified 28 STs among 76 E. coli isolates and MLST showed abundant genetic diversity in the E. coli. Similar to other studies in the world (van Hout et al. 2020; Yasir et al. 2018), ST131 was the predominant ST. Moreover, we found that the predominant ESBLs type in the B2-ST131 (phylogenetic group-sequence type) isolates was CTX-M-14, rather than CTX-M-15 as reported in Australia (Harris et al. 2018), indicating an association of distinct CTX-M types with different settings due to various modes of transmission. In our study, ST69 was the most prevalent among non-ESBLs-producers, while one study from Netherlands showed that ST73 was the most common sequence type among non-ESBLs-producing isolates (van Hout et al. 2020). This proved that the distribution of STs of E. coli vary significantly by region. Our study found that males accounted for $60.0 \%$ of patients infected with ST131 and females accounted for $83.3 \%$ of patients infected with ST69, consistent with a Canadian study (Holland et al. 2020). The reasons for genders differences in E. coli ST types are unclear and require further study.

\section{Conclusions}

In conclusion, data for this article described the drug sensitivity and molecular epidemiology of E. coli in patients with BSIs in Shanxi, China. We found that E. coli were highly resistant to commonly used antibiotics, except for carbapenems, piperacillin-tazobactam, amikacin and tigecycline. The B2-ST131 and D-ST69 clonal groups were the most common clinically relevant genotypes. Phylogenetic analysis showed genetic diversity among E. coli isolates. Better monitoring of the epidemiology of $E$. coli bacteremia is needed to develop and implement effective prevention strategies.

\section{Acknowledgements}

We would like to thank Yu Zhang of Shanxi Medical University for her help in our experiment. 


\section{References}

247

248

249

250

251

252

253

254

255

256

257

258

259

260

261

262

263

264

265

266

267

268

269

270

271

272

273

274

275

276

277

278

279

280

281

282

283

284

285

286
Bi W, Li B, Song J, Hong Y, Zhang X, Liu H, Lu H, Zhou T, and Cao J. 2017. Antimicrobial susceptibility and mechanisms of fosfomycin resistance in extended-spectrum $\beta$-lactamase-producing Escherichia coli strains from urinary tract infections in Wenzhou, China. Int J Antimicrob Agents.

Bou-Antoun S, Davies J, Guy R, Johnson AP, Sheridan EA, and Hope RJ. 2016. Descriptive epidemiology of Escherichia coli bacteraemia in England, April 2012 to March 2014. Euro Surveill.

Clermont O, Bonacorsi S, and Bingen E. 2000. Rapid and simple determination of the Escherichia coli phylogenetic group. Appl Environ Microbiol 66:4555-4558. 10.1128/aem.66.10.45554558.2000Diekema DJ, Hsueh P-R, Mendes RE, Pfaller MA, Rolston KV, Sader HS, and Jones RN. 2019. The Microbiology of Bloodstream Infection: 20-Year Trends from the SENTRY Antimicrobial Surveillance Program. Antimicrob Agents Chemother.

CLSI (2019) CLSI performance standards for antimicrobial susceptibility testing; twenty-ninth informational supplement. Clinical and Laboratory Standards Institute, Wayne, PACLSI document M100-S29. 2019

EUCAST (2019). The European Committee on Antimicrobial Susceptibility Testing; Breakpoint Tables for Interpretation of MICs and Zone Diameters, version 9.0, 2019.

Goto M, and Al-Hasan MN. 2013. Overall burden of bloodstream infection and nosocomial bloodstream infection in North America and Europe. Clin Microbiol Infect.

Harris PNA, Ben Zakour NL, Roberts LW, Wailan AM, Zowawi HM, Tambyah PA, Lye DC, Jureen R, Lee TH, Yin M, Izharuddin E, Looke D, Runnegar N, Rogers B, Bhally H, Crowe A, Schembri MA, Beatson SA, Paterson DL, and investigators MT. 2018. Whole genome analysis of cephalosporin-resistant Escherichia coli from bloodstream infections in Australia, New Zealand and Singapore: high prevalence of CMY-2 producers and ST131 carrying blaCTX-M-15 and blaCTX-M-27. J Antimicrob Chemother.

Holland MS, Nobrega D, Peirano G, Naugler C, Church DL, and Pitout JDD. 2020. Molecular epidemiology of Escherichia coli causing bloodstream infections in a centralized Canadian region: a population-based surveillance study. Clin Microbiol Infect.

Hu F, Guo Y, Yang Y, Zheng Y, Wu S, Jiang X, Zhu D, Wang F, and Group CASNS. 2019. Resistance reported from China antimicrobial surveillance network (CHINET) in 2018. Eur J Clin Microbiol Infect Dis.

Hu X, Gou J, Guo X, Cao Z, Li Y, Jiao H, He X, Ren Y, and Tian F. 2018. Genetic contexts related to the diffusion of plasmid-mediated CTX-M-55 extended-spectrum beta-lactamase isolated from Enterobacteriaceae in China. Ann Clin Microbiol Antimicrob.

Huh K, Chung DR, Ha YE, Ko J-H, Kim S-H, Kim M-J, Huh HJ, Lee NY, Cho SY, Kang C-I, Peck KR, Song J-H, and investigators KARSN. 2020. Impact of difficult-to-treat resistance in gram-negative bacteremia on mortality: retrospective analysis of nationwide surveillance data. Clin Infect Dis.

Hung PN, Quyet D, Thanh KC, Pho DC, Tien TV, Dung QA, Linh DD, Tan HT, Dinh TC, Bac ND, and Nam LV. 2019. Escherichia coliAntibiotic Resistance Profile and Diversity of Subtypes Genes in Causing Bloodstream Infection in Northern Vietnam. Open Access Maced J Med Sci.

Javed S, Mirani ZA, and Pirzada ZA. 2021. Phylogenetic Group B2 Expressed Significant Biofilm Formation among Drug Resistant Uropathogenic Escherichia coli. Libyan J Med 16:1845444. $10.1080 / 19932820.2020 .1845444$

Peer) reviewing PDF | (2021:04:59823:2:0:NEW 30 Sep 2021) 
Kern WV, and Rieg S. 2019. Burden of bacterial bloodstream infection-a brief update on epidemiology and significance of multidrug-resistant pathogens. Clin Microbiol Infect.

Komatsu Y, Kasahara K, Inoue T, Lee S-T, Muratani T, Yano H, Kirita T, and Mikasa K. 2018. Molecular epidemiology and clinical features of extended-spectrum beta-lactamase- or carbapenemaseproducing Escherichia coli bacteremia in Japan. PLoS One.

Liao $K$, Chen $Y$, Wang $M$, Guo $P$, Yang $Q$, Ni $Y$, Yu $Y$, Hu B, Sun Z, Huang $W$, Wang $Y$, Wu A, Feng $X$, Luo $Y$, Hu Z, Chu Y, Chen S, Cao B, Su J, Gui B, Duan Q, Zhang S, Shao H, Kong H, and Xu Y. 2017. Molecular characteristics of extended-spectrum $\beta$-lactamase-producing Escherichia coli and Klebsiella pneumoniae causing intra-abdominal infections from 9 tertiary hospitals in China. Diagn Microbiol Infect Dis.

Ma J, Li N, Liu Y, Wang C, Liu X, Chen S, Xie X, Gan S, Wang M, Cao W, Wang F, Liu Y, Wan D, Sun L, and Sun H. 2017. Antimicrobial resistance patterns, clinical features, and risk factors for septic shock and death of nosocomial $E$ coli bacteremia in adult patients with hematological disease: A monocenter retrospective study in China. Medicine (Baltimore).

Marchello CS, Dale AP, Pisharody S, Rubach MP, and Crump JA. 2019. A Systematic Review and Metaanalysis of the Prevalence of Community-Onset Bloodstream Infections among Hospitalized Patients in Africa and Asia. Antimicrob Agents Chemother.

Mehl A, Asvold BO, Lydersen S, Paulsen J, Solligard E, Damas JK, Harthug S, and Edna TH. 2017. Burden of bloodstream infection in an area of Mid-Norway 2002-2013: a prospective population-based observational study. BMC Infect Dis 17:205. 10.1186/s12879-017-2291-2

Nepal K, Pant ND, Neupane B, Belbase A, Baidhya R, Shrestha RK, Lekhak B, Bhatta DR, and Jha B. 2017. Extended spectrum beta-lactamase and metallo beta-lactamase production among Escherichia coli and Klebsiella pneumoniae isolated from different clinical samples in a tertiary care hospital in Kathmandu, Nepal. Ann Clin Microbiol Antimicrob.

Parajuli NP, Acharya SP, Mishra SK, Parajuli K, Rijal BP, and Pokhrel BM. 2017. High burden of antimicrobial resistance among gram negative bacteria causing healthcare associated infections in a critical care unit of Nepal. Antimicrob Resist Infect Control 6:67. 10.1186/s13756-017-0222-z

Ranjan A, Shaik S, Nandanwar N, Hussain A, Tiwari SK, Semmler T, Jadhav S, Wieler LH, Alam M, Colwell RR, and Ahmed N. 2017. Escherichia coliComparative Genomics of Isolated from Skin and Soft Tissue and Other Extraintestinal Infections. mBio.

Rohde AM, Zweigner J, Wiese-Posselt M, Schwab F, Behnke M, Kola A, Schröder W, Peter S, Tacconelli E, Wille T, Feihl S, Querbach C, Gebhardt F, Gölz H, Schneider C, Mischnik A, Vehreschild MJGT, Seifert H, Kern WV, Gastmeier P, and Hamprecht A. 2020. Prevalence of third-generation cephalosporin-resistant Enterobacterales colonization on hospital admission and ESBL genotypespecific risk factors: a cross-sectional study in six German university hospitals. J Antimicrob Chemother.

Sader HS, Castanheira M, Streit JM, and Flamm RK. 2019. Frequency of occurrence and antimicrobial susceptibility of bacteria isolated from patients hospitalized with bloodstream infections in United States medical centers (2015-2017). Diagn Microbiol Infect Dis.

Tian L, Zhang Z, and Sun Z. 2019. Antimicrobial resistance trends in bloodstream infections at a large teaching hospital in China: a 20-year surveillance study (1998-2017). Antimicrob Resist Infect Control.

Tsuzuki S, Matsunaga N, Yahara K, Gu Y, Hayakawa K, Hirabayashi A, Kajihara T, Sugai M, Shibayama K, and Ohmagari N. 2020. National trend of blood-stream infection attributable deaths caused by Staphylococcus aureus and Escherichia coli in Japan. J Infect Chemother.

van Hout D, Verschuuren TD, Bruijning-Verhagen PCJ, Bosch T, Schürch AC, Willems RJL, Bonten MJM, and Kluytmans JAJW. 2020. Extended-spectrum beta-lactamase (ESBL)-producing and non-ESBL-

Peer] reviewing PDF | (2021:04:59823:2:0:NEW 30 Sep 2021) 
producing Escherichia coli isolates causing bacteremia in the Netherlands (2014 - 2016) differ in clonal distribution, antimicrobial resistance gene and virulence gene content. PLoS One.

Vihta K-D, Stoesser N, Llewelyn MJ, Quan TP, Davies T, Fawcett NJ, Dunn L, Jeffery K, Butler CC, Hayward G, Andersson M, Morgan M, Oakley S, Mason A, Hopkins S, Wyllie DH, Crook DW, Wilcox MH, Johnson AP, Peto TEA, and Walker AS. 2018. Trends over time in Escherichia coli bloodstream infections, urinary tract infections, and antibiotic susceptibilities in Oxfordshire, UK, 1998-2016: a study of electronic health records. Lancet Infect Dis.

Wang S, Zhao SY, Xiao SZ, Gu FF, Liu QZ, Tang J, Guo XK, Ni YX, and Han LZ. 2016. Antimicrobial Resistance and Molecular Epidemiology of Escherichia coli Causing Bloodstream Infections in Three Hospitals in Shanghai, China. PLoS One 11:e0147740. 10.1371/journal.pone.0147740

Xiao T, Wu Z, Shi Q, Zhang X, Zhou Y, Yu X, and Xiao Y. 2019a. A retrospective analysis of risk factors and outcomes in patients with extended-spectrum beta-lactamase-producing Escherichia coli bloodstream infections. J Glob Antimicrob Resist.

Xiao T, Yang K, Zhou Y, Zhang S, Ji J, Ying C, Shen P, and Xiao Y. 2019b. Escherichia coliRisk factors and outcomes in non-transplant patients with extended-spectrum beta-lactamase-producing bacteremia: a retrospective study from 2013 to 2016. Antimicrob Resist Infect Control.

Yasir M, Ajlan AM, Shakil S, Jiman-Fatani AA, Almasaudi SB, Farman M, Baazeem ZM, Baabdullah R, Alawi M, Al-Abdullah N, Ismaeel NA, Shukri HA, and Azhar El. 2018. Molecular characterization, antimicrobial resistance and clinico-bioinformatics approaches to address the problem of extended-spectrum $\beta$-lactamase-producing Escherichia coli in western Saudi Arabia. Sci Rep.

Yoon E-J, Choi MH, Park YS, Lee HS, Kim D, Lee H, Shin KS, Shin JH, Uh Y, Kim YA, Shin JH, and Jeong SH. 2018. Impact of host-pathogen-treatment tripartite components on early mortality of patients with Escherichia coli bloodstream infection: Prospective observational study. EBioMedicine.

Zhang C-Z, Ding X-M, Lin X-L, Sun R-Y, Lu Y-W, Cai R-M, Webber MA, Ding H-Z, and Jiang H-X. 2019. blaThe Emergence of Chromosomally Located in From Foodborne Animals in China. Front Microbiol.

Zhao SY, Wang YC, Xiao SZ, Jiang XF, Guo XK, Ni YX, and Han LZ. 2015. Drug susceptibility and molecular epidemiology of Escherichia coli in bloodstream infections in Shanghai, China, 2011-2013. Infect Dis (Lond) 47:310-318. 10.3109/00365548.2014.990509

Zou H, Zheng B, Sun M, Ottoson J, Li Y, Berglund B, Chi X, Ji X, Li X, Stålsby Lundborg C, and Nilsson LE. 2019. Escherichia coliEvaluating Dissemination Mechanisms of Antibiotic-Resistant Bacteria in Rural Environments in China by Using CTX-M-Producing as an Indicator. Microb Drug Resist.

Peer) reviewing PDF | (2021:04:59823:2:0:NEW 30 Sep 2021) 
Figure 1

Distribution of phylogenetic groups 


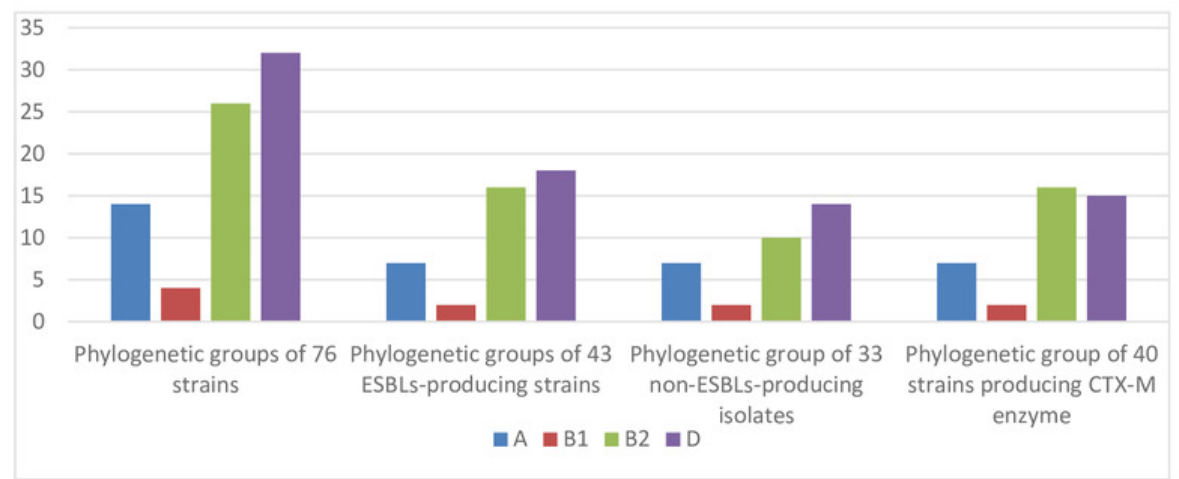

Fig. 1. Distribution of phylogenetic groups 


\section{Table $\mathbf{1}$ (on next page)}

Sequences of primers for resistance genes PCR amplification 
Table 1. Sequences of primers for resistance genes PCR amplification.

\begin{tabular}{|c|c|c|c|}
\hline Gene & Primers a & Primer Sequences (5'-3') & Expected Amplicon Size (bp) \\
\hline \multirow[t]{2}{*}{ TEM } & F & ATAAAATTCTTGAAGACGAAA & 1080 \\
\hline & $\mathrm{R}$ & GACAGTTACCAATGCTTAATC & \\
\hline \multirow[t]{2}{*}{ SHV } & $\mathrm{F}$ & TGGTTATGCGTTATATTCGCC & 865 \\
\hline & $\mathrm{R}$ & GGTTAGCGTTGCCAGTGCT & \\
\hline \multirow[t]{2}{*}{ CTX-M-1 } & $\mathrm{F}$ & AAAAATCACTGCGCCAGTTC & 415 \\
\hline & $\mathrm{R}$ & AGCTTATTCATCGCCACGTT & \\
\hline \multirow[t]{2}{*}{ CTX-M-2 } & $\mathrm{F}$ & CGACGCTACCCCTGCTATT & 552 \\
\hline & $\mathrm{R}$ & CCAGCGTCAGATTTTTCAGG & \\
\hline \multirow[t]{2}{*}{ CTX-M-8 } & $\mathrm{F}$ & TCGCGTTAAGCGGATGATGC & 666 \\
\hline & $\mathrm{R}$ & AACCCACGATGTGGGTAGC & \\
\hline \multirow[t]{2}{*}{ CTX-M-9 } & $\mathrm{F}$ & CAAAGAGAGTGCAACGGATG & 205 \\
\hline & $\mathrm{R}$ & ATTGGAAAGCGTTCATCACC & \\
\hline \multirow[t]{2}{*}{ CTX-M-25 } & $\mathrm{F}$ & GCACGATGACATTCGGG & 327 \\
\hline & $\mathrm{R}$ & AACCCACGATGTGGGTAGC & \\
\hline \multirow[t]{2}{*}{ OXA-1 } & $\mathrm{F}$ & CTGTTGTTTGGGTTTCGCAAG & 440 \\
\hline & $\mathrm{R}$ & CTTGGCTTTTATGCTTGATG & \\
\hline \multirow[t]{2}{*}{ OXA-2 } & $\mathrm{F}$ & CAGGCGCYGTTCGYGATGAGTT & 233 \\
\hline & $\mathrm{R}$ & GCCYTCTATCCAGTAATCGCC & \\
\hline \multirow[t]{2}{*}{ OXA-10 } & $\mathrm{F}$ & GTCTTTCRAGTACGGCATTA & 822 \\
\hline & $\mathrm{R}$ & GATTTTCTTAGCGGCAACTTA & \\
\hline \multirow[t]{2}{*}{ VEB } & $\mathrm{F}$ & GCGGTAATTTAACCAGA & 961 \\
\hline & $\mathrm{R}$ & GCCTATGAGCCAGTGTTC & \\
\hline \multirow[t]{2}{*}{ GES } & $\mathrm{F}$ & ATGCGCTTCATTCACGCAC & 846 \\
\hline & $\mathrm{R}$ & CTATTTGTCCGTGCTCAGG & \\
\hline \multirow[t]{2}{*}{ PER } & $\mathrm{F}$ & AGTCAGCGGCTTAGATA & 978 \\
\hline & $\mathrm{R}$ & CGTATGAAAAGGACAATC & \\
\hline
\end{tabular}

\footnotetext{
2 a Primer, The ' $F$ ' meant the forward primer and the ' $R$ ' meant the reverse primer

3

4

5

6

7

8

9

10

11

12

13
} 
Table 2 (on next page)

Primers sequences of the housekeeping genes of $E$. coli 


\begin{tabular}{|c|c|c|c|}
\hline Gene & Primers a & Primer Sequences (5'-3') & $\begin{array}{l}\text { Expected Amplicon } \\
\text { Size (bp) }\end{array}$ \\
\hline \multirow[t]{2}{*}{$a d K$} & $\mathrm{~F}$ & ATTCTGCTTGGCGCTCCGGG & 536 \\
\hline & $\mathrm{R}$ & CCGTCAACTTTCGCGTATTT & \\
\hline \multirow[t]{2}{*}{ fum $C$} & $\mathrm{~F}$ & TCACAGGTCGCCAGCGCTTC & 469 \\
\hline & $\mathrm{R}$ & TCCCGGCAGATAAGCTGTGG & \\
\hline \multirow[t]{2}{*}{$\operatorname{gyr} B$} & $\mathrm{~F}$ & TCGGCGACACGGATGACGGC & 460 \\
\hline & $\mathrm{R}$ & GTCCATGTAGGCGTTCAGGG & \\
\hline \multirow[t]{2}{*}{$i c d$} & $\mathrm{~F}$ & ATGGAAAGTAAAGTAGTTGTTCCGGCACA & 518 \\
\hline & $\mathrm{R}$ & GGACGCAGCAGGATCTGTT & \\
\hline \multirow[t]{2}{*}{$m d h$} & $\mathrm{~F}$ & AGCGCGTTCTGTTCAAATGC & 452 \\
\hline & $\mathrm{R}$ & CAGGTTCAGAACTCTCTCTGT & \\
\hline \multirow[t]{2}{*}{ purA } & $\mathrm{F}$ & TCGGTAACGGTGTTGTGCTG & 478 \\
\hline & $\mathrm{R}$ & CATACGGTAAGCCACGCAGA & \\
\hline \multirow[t]{2}{*}{ recA } & $\mathrm{F}$ & CGCATTCGCTTTACCCTGACC & 510 \\
\hline & $\mathrm{R}$ & AGCGTGAAGGTAAAACCTGTG & \\
\hline
\end{tabular}

2 a Primer, The ' $F$ ' meant the forward primer and the ' $\mathrm{R}$ ' meant the reverse primer 3 
Table 3 (on next page)

Rates of antimicrobial resistance among $E$. coli bloodstream isolates 


\begin{tabular}{|c|c|c|c|c|}
\hline \multirow[t]{2}{*}{ Antimicrobial agents } & \multicolumn{3}{|c|}{ Number of isolates $(\%)$} & \multirow[t]{2}{*}{$p$} \\
\hline & Total $(\mathrm{n}=76)$ & $\operatorname{ESBLs}(n=43)$ & non-ESBLs $(\mathrm{n}=33)$ & \\
\hline AMP & $69(90.7)$ & $43(100.0)$ & $26(78.7)$ & 0.002 \\
\hline $\mathrm{AMC}$ & $5(6.5)$ & $4(9.3)$ & $1(3.0)$ & 0.274 \\
\hline TZP & 0 & 0 & 0 & - \\
\hline CFZ & $50(65.7)$ & $43(100.0)$ & $7(21.2)$ & $<0.0001$ \\
\hline FOX & $11(14.4)$ & $9(20.9)$ & $2(6.0)$ & 0.068 \\
\hline $\mathrm{CRO}$ & $43(56.5)$ & $43(100.0)$ & 0 & $<0.0001$ \\
\hline $\mathrm{CEF}$ & $11(14.4)$ & $11(25.5)$ & 0 & 0.002 \\
\hline CTX & $43(56.5)$ & $43(100.0)$ & 0 & $<0.0001$ \\
\hline CPS & $1(1.3)$ & $1(2.3)$ & 0 & 0.378 \\
\hline ATM & $28(36.8)$ & $28(65.1)$ & 0 & $<0.0001$ \\
\hline ETP & 0 & 0 & 0 & - \\
\hline IPM & 0 & 0 & 0 & - \\
\hline MEM & 0 & 0 & 0 & - \\
\hline $\mathrm{AK}$ & 0 & 0 & 0 & - \\
\hline GM & $40(52.6)$ & $26(60.4)$ & $14(42.4)$ & 0.118 \\
\hline TOB & $3(3.9)$ & $3(6.9)$ & 0 & 0.122 \\
\hline CIP & $53(69.7)$ & $37(86.0)$ & $16(48.4)$ & $<0.0001$ \\
\hline LEV & $48(63.1)$ & $36(83.7)$ & $12(36.3)$ & $<0.0001$ \\
\hline TGC & 0 & 0 & 0 & - \\
\hline SXT & $38(50.0)$ & $23(53.4)$ & $15(45.5)$ & 0.488 \\
\hline
\end{tabular}


2 AMP, ampicillin; AMC, Amoxicillin-clavulanate potassium; TZP, piperacillin-tazobactam; CFZ, Cefazolin; 3 FOX, Cefoxitin; CRO, ceftriaxone; CEF, cefepime; CTX, Cefotaxime; CPS, Cefoperazone-sulbactam; ATM, 4 aztreonam; ETP, ertapenem; IPM, imipenem; MEM, meropenem; AK, amikacin; GM, gentamicin; ; TOB, 5 tobramycin; CIP, ciprofloxacin; LEV, levofloxacin; TGC, Tigecycline; SXT, trimethoprim-sulfamethoxazole. 
Table 4 (on next page)

Phylogenetic groups and genotypes in MLST of $76 \mathrm{E}$. coli isolates 
Table 4. Phylogenetic groups and genotypes in MLST of 76 E. coli isolates

\begin{tabular}{|c|c|c|c|}
\hline Sequence Type & $\begin{array}{c}\text { Phylogenetic } \\
\text { groups }\end{array}$ & Resistance determinants & $\begin{array}{l}\text { No. of } \\
\text { isolates }\end{array}$ \\
\hline \multirow[t]{6}{*}{ ST131 } & $\mathrm{B} 2$ & TEM-1, CTX-M-14 & 5 \\
\hline & B2 & CTX-M-14 & 3 \\
\hline & B2 & - & 3 \\
\hline & B2 & CTX-M-15,CTX-M-14, OXA-1 & 2 \\
\hline & B2 & CTX-M-55, CTX-M-14 & 1 \\
\hline & B2 & TEM-1, CTX-M-15, OXA-1 & 1 \\
\hline \multirow[t]{3}{*}{ ST69 } & $\mathrm{D}$ & - & 8 \\
\hline & $\mathrm{D}$ & TEM-1 & 3 \\
\hline & $\mathrm{D}$ & CTX-M-14 & 1 \\
\hline \multirow[t]{5}{*}{ ST38 } & $\mathrm{D}$ & - & 2 \\
\hline & $\mathrm{D}$ & CTX-M-14 & 1 \\
\hline & $\mathrm{D}$ & CTX-M-15 & 1 \\
\hline & $\mathrm{D}$ & TEM-1, CTX-M-14 & 1 \\
\hline & $\mathrm{D}$ & CTX-M-15, CTX-M-14 & 1 \\
\hline \multirow[t]{4}{*}{ ST1193 } & B2 & - & 2 \\
\hline & B2 & CTX-M-14 & 1 \\
\hline & B2 & TEM-1, CTX-M-55 & 1 \\
\hline & $\mathrm{B} 2$ & TEM-1, CTX-M-15 & 1 \\
\hline ST73 & $\mathrm{B} 2$ & - & 4 \\
\hline ST648 & $\mathrm{D}$ & - & 2 \\
\hline
\end{tabular}




\begin{tabular}{|c|c|c|c|}
\hline & $\mathrm{D}$ & CTX-M-14 & 1 \\
\hline & $\mathrm{D}$ & CTX-M-55 & 1 \\
\hline \multirow[t]{2}{*}{ ST46 } & A & CTX-M-14 & 2 \\
\hline & A & CTX-M-55 & 1 \\
\hline ST405 & $\mathrm{D}$ & TEM-1, CTX-M-14 & 3 \\
\hline \multirow[t]{2}{*}{ ST117 } & $\mathrm{D}$ & TEM-1, CTX-M-14 & 1 \\
\hline & $\mathrm{D}$ & TEM-1, CTX-M-55 & 1 \\
\hline ST450 & A & - & 2 \\
\hline \multirow[t]{2}{*}{ ST2003 } & $\mathrm{D}$ & TEM-1, CTX-M-14 & 1 \\
\hline & $\mathrm{D}$ & CTX-M-14 & 1 \\
\hline ST2448 & $\mathrm{B} 1$ & - & 2 \\
\hline ST44 & A & CTX-M-15,OXA-1 & 1 \\
\hline ST10 & A & - & 1 \\
\hline ST410 & A & TEM-1, СТХ-M-15, OXA-1 & 1 \\
\hline ST457 & $\mathrm{D}$ & TEM-1, CTX-M-15 & 1 \\
\hline ST2179 & $\mathrm{B} 1$ & CTX-M-14,OXA-1 & 1 \\
\hline ST393 & $\mathrm{D}$ & - & 1 \\
\hline ST1163 & $\mathrm{D}$ & - & 1 \\
\hline ST155 & $\mathrm{B} 1$ & CTX-M-14 & 1 \\
\hline ST773 & $\mathrm{A}$ & - & 1 \\
\hline ST1284 & A & CTX-M-15,OXA-1 & 1 \\
\hline ST95 & B2 & - & 1 \\
\hline ST493 & B2 & CTX-M-55 & 1 \\
\hline
\end{tabular}




\begin{tabular}{|c|c|c|c|}
\hline ST542 & $\mathrm{A}$ & - & 1 \\
\hline ST409 & $\mathrm{A}$ & - & 1 \\
\hline ST167 & A & - & 1 \\
\hline ST4503 & A & CTX-M-14 & 1 \\
\hline
\end{tabular}

2

3 